\title{
CALVINO E IL CINEMA: LA VOCE, LO SGUARDO, LA DISTANZA.
}

Uno degli scritti più suggestivi e relativamente meno noti cli Calvino si intitola "Il silenzio e la città." Appárve nel volume di Giovanni Fánelli intitolato Firenze perduta: L immagine di Firenze nei 120 dipinti di labio Borbottoni (1820-1901). edito da Franco Maria Ricci nel 1982. Devo la scoperta di questo testo al traduttore di Calvino, William Weaver, che lo lesse ad alta roce durante un convegno su Calvino al quale partecipammo insieme qualche anno fa all'U 'niversità della Cálifornia a Davis. Ascoltandolo lo trovai stranamente commovente. Leggendolo poi accanto alle riproduzioni dei quadri di Borbottoni (un artista di cui non avevo mai sentito parlare prima), mi colpí il contrasto tra le immagini estremamente statiche (henché suggestive e delicatamente poetiche), e il senso di movimento, o meglio di visione in movimento, che scaturiva dal testo di Calvino. Il testo inizia cosí: "Il silenzio entrò nella città di primo pomeriggio. Sinfilò per le porte turrite e merlate, occupò le logge arcata per arcata, s’incanalò per le strade a ridosso delle mura, s’addossò agli spalti, ricolmò gli scannafossi". I E' come se Calvino avesse letteralmente animato il silenzio del pittore, dando vita alle immagini e mettendole in movimento, pur mantenendo allo stesso tempo il senso di una mancanza assoluta di voci e di suoni. Leggenclo il testo si ha l'impressione di guardare un breve film muto surrealista.

La passione di Calvino per le arti visive è ben nota. Nelle sue opere letterarie viene sempre privilegiato lo sguardo, l'atto di vedere, guardare, osservare, e la fenomenologia della visione in tutti i suoi aspetti. Ciò lo rende forse il più "visivo" di tutti gli scrittori italiani contemporanei. Lo stesso Calvino, nella lezione per l'università di Harvard sulla "Visibilità" che divenne poi uno dei Six Memos for the Next Millenmimm (noti nella versione italiana come Lezioni americane), fa notare che allorigine di ognuno dei suoi racconti c'è un’immagine visivá un uomo tagliato a metà. un ragalzzo che si arrampica su un albero. un'armatura vuota che si muove e parla. Ma lossessione visiva di Calvino non si limita alle immagini iniziali: anche l'organizzazione della narrazione, le simmetrie che la caratterizzano e perfino il suo svolgersi concettuale tendono ad essere ordinati visivamente (basti pen- 
sare a Il castello dei destini incrociati o a Le città imusibili), come se le storie di Calvino fossero destinate prima di tutto a essere viste, visualizzate anziché "lette," ascoltate e afferrate o comprese in altro modo. Le cosmicomiche e Palomar offrono esempi illuminanti di questa tecnica di narrare per immagini. La tecnica visiva di Calvino è basata sulla manipolazione della distanza. Tutti ricordano il modo spassionato e vagamente comico con cui il signor Palomar guarda il seno nudo della bagnante da una prospettiva estremamente ravricinata, come se i suoi occhi, simili a una cinepresa, fossero in grado di effettuare un potente movimento di zoom. Come ha osservato Antonio Costa, la scansione narrativa di Calvino e le sue tecniche di inquadratura appaiono spesso spiccatamente cinematiche. 2 Il modo di guardare di Calvino è in effetti per molti versi analogo a quello del cinema, specialmente il cinema hollywoodiano degli anni 30 di cui Calvino si nutriva giornalmente da ragazzo a San Remo prima della seconda guerra mondiale. Calvino usciva di casá nel pomeriggio e (all'insaputa dei genitori) andava al cinema, a veclere clue, a volte tre film quasi tutti i giorni. Secondo quanto Calvino ci racconta nel suo saggio clel 1971, "Autobiografia di uno spettatore", anclare al cinema aveva il gusto di un piacere proibito. ${ }^{3}$ C'è da pensare che il cinema abbia contribuito in modo determinante a formare l'immaginazione di Calvino, forse più dei libri letti o clegli amati fumetti del Corriere dei piccoli. Perciò non sorprende che il primo testo in assoluto pubblicato da Calvino sia stato probabilmente un pezzo sul cinema intitolato "Scene e schermi" siglato "I. c." e apparso sul Giomale di Imperia il 7 luglio 1941, ${ }^{i}$ e che Le città invisibili fosse stato concepito inizialmente da Calvino come sceneggiatura per un film (che non fu mai realizzato). ${ }^{5}$

Al cinema Calvino vedeva tutto, compresi i film fascisti di quel periodo, ma i film che lasciarono le tracce più profonde, diventando per lui sinonimi del cinema stesso, furono i film hollywoodiani con le grandi stelle come Spencer Tracy, Clark Gable, Gary Cooper, William Powell e soprattutto gli indimenticabili corpi e visi di Jean Harlow, Marlene Dietrich, Barbara Stanwick e Jean Crawforcl. Dico "corpi e visi" perché naturalmente questi film erano cloppiati in italiano. Calvino ha delle cose interessanti da dire nella sua "Autobiografia di uno spettatore" sugli effetti perturbanti del doppiaggio. Le voci italiane gli sembravano completamente e fastidiosamente estranee alle immagini sullo schermo, e come spettatore egli tendeva ad ignorarle facendo finta di non sentirle (in parte perché sapeva che il dialogo originale veniva spesso contraffatto dalla censura). Il fascino del cinema per Calvino aveva poco á che vedere con la voce e con le parole: era soprattutto un fascino visivo e in un certo senso il cinema per lui rimase per sem- 
pre muto, un esperienza essenzialmente di sguardi. La storia la si coglieva attraverso le immagini che si muovevano proiettate sullo schermo; della voce e del clialogo si poteva anche fare a meno. Le immagini in bianco e nero dei corpi e visi delle stelle di Hollywood, soprattutto le donne, erano in un certo senso anchesse incorporee e astratte, puri segni sulla superficie dello schermo: "Anche la più carnale delle americane di allora, la biondoplatino Jean Harlow, era resa irreale clal biancore abbagliante della pelle. Nel bianco-e-nero la forza del bianco operava una trasfigurazione dei visi femminili, clelle gambe, delle spalle e scollature, faceval di Marlene Dietrich non l'oggetto immediato del desiderio ma il desiderio stesso come essenza extraterrestre"( $p$. 34). Attraverso la rimozione calviniana della voce, la normale distanza dello spettatore dallo schermo risulta perciò raddoppiata, moltiplicata al punto da far sembrare che le immagini emanino da corpi celesti e che il desiderio sia una sostanza purificata ed ineffabile. 6

I primi film che Calvino vide da bambino erano film muti. Erano per la maggior parte film educativi attentamente selezionati dai genitori, che avevano un proiettore in casa. La madre di Calvino permetteva che si vedessero alcune comiche di Harold Lloyd e di Charlie Chaplin, ma al seconclo preferiva il primo, perché a suo dire Charlot era troppo maleducato. ${ }^{-}$In un intervista del 1981 con Lietta Tornabuoni, Calvino ricorda di essere andato al cinema a vedere per la prima volta clue film "parlati" accompagnato da sua madre. Si trattava di due pseudo-documentari, Afirica parla e Trader Horn. Contenevano scene di violenza e di tortura "inflitta clai selvaggi agli esploratori" e la madre di Calvino, giuclicando i film "nocivi al sistema nerroso", si alzó e portò via Calvino prima della fine. ${ }^{8}$ La prima esperienza calviniana del "parlato" cinematografico arvenne quindi sotto il segno del "primitivo," della violenza, e della proibizione materna. Il senso di disagio che Calvino sentí sempre nei confronti della parola e della voce è ben noto. A volte Calvino si trovava letteralmente a balbettare come un bambino in cerca clelle parole da dire, mentre invece amava molto disegnare, scárabocchiare, fare diagrammi e schizzi (era un grande ammiratore di Saul Steinberg), e naturalmente scrivere. Lál parola orale, dettá a voce alta, era invece addirittura repellente per Calvino. In un colloquio del 1973 con Ferdinando Camon (condotto per la maggior parte attraverso uno scambio epistolare), Calvino si espresse in termini espliciti e (per lui di solito cosi riservato) stranamente "forti" in proposito: "Ho sempre avuto un certo disgusto per la parola parlata . . . un disgusto fondamentale per lá parola, per questa cosa che esce dalla bocca, informe, molle molle"? $\mathrm{E}^{\prime}$ interessante notare come per Calvino la parola parlata sia intimamente connessa con il corpo. 
con la bocca e la lingua, e quindi con l'oralità in senso fisico che risale all'esperienza "primitiva" del poppante, e a quello che Freud chiama il piacere della "fase orale." Tale piacere secondo Freud diviene successivamente, nel distanziamento obbligatorio del bambino dal corpo materno (messo in vigore dalla madre stessa), oggetto di proibizione e censura e quindi di "disgusto." 10 Il piacere orale viene distanziato, rimosso, e spostato su altri oggetti. Per quanto disgustosa, la voce sembra quindi mantenere per Calvino un aspetto perturbante. Per dare piacere, un film per Calvino deve comunque essere muto, o essere "reso" muto (il doppiaggio fascista serviva a legittimare questo desiderio di silenzio). Il cinema, che nella sua purezza originaria era in effetti muto, è una forma di spettacolo che (contrariamente al teatro, che non può fare a meno (lella voce) comunica essenzialmente per immagini. Nel prediligere nel cinema il silenzio, e la purezza visiva delle immagini, Calvino si avvicina a Pirandello (che in un famoso saggio deprecò l'invenzione del parlato) e a registi come Bergman, Antonioni e Bresson.

La qualità perturbante della voce e del parlato per Calvino, e il suo clesiderio di non sentirli o di distanziarli, può avere anche una spiegazione politica. Come la letteratura americana, anche il cinema americano negli anni '30 rappresentava una dimensione quasi mitica cli alterità, di apertura e di libertà per chi viveva nella realtà autarchica e soffocante dell'Italia fascista. Quelle voci italiane che doppiavano gli attori americani (cambiandone le parole secondo criteri dettati dal regime) rapresentavano quasi una fascistizzazione e quindi una corruzione del cinema americano. Anche i grandi film francesi clegli anni ' 30 per Calvino erano associabili con il cinema americano in quanto "altri" rispetto al fascismo, e permettevano di fantasticare di una vita e un mondo diversi. Dei film italiani visti in quel periodo Calvino dice solo (nell' "Autobiografia di uno spettatore") che non valeva la pena di ricordarli. Tuttavia, in un brano suggestivo dello stesso saggio, Calvino ricorda come proprio quelle voci italiane, false e presumibilmente odiose, quando le udiva emergere dalle sale cinematografiche davanti a cui passava passeggiando di pomeriggio, lo attraessero come un canto delle sirene, invitandolo a entrare e ad abbandanarsi alla seduzione del cinema.

Quando andava di nascosto al cinema nel pomeriggio a San Remo, Calvino di solito si trovava solo nella sala buia, e metteva le gambe a cavalcioni della poltrona davanti alla sua. Tutti i suoi amici erano fuori a giocare a pallone o a fare su e giù per il corso nel rituale della passeggiata e del corteggiamento adolescenziale, fatto di occhiate e chiacchiere. Calvino preferiva il buio accogliente e la solitudine della sala cinematografica, e l'erotismo dello spettatore. Quest'erotismo dello spettatore venne a costituire 
un aspetto primario della sensibilità di Calvino scrittore, contribuendo a clare forma e a controllare l'intera sua produzione letteraria e critica. Lá distanzá necessaria di cui Cálvino parla cosi spesso, e che è stata ripetutamente interpretata dai critici come sintomo del suo intellettualismo, della suá presunta freddezza e mancanza di passione, di un suo scontroso Cartesianesimo, è soprattutto dá vedere invece come la distanza che separa lo spetatore dalle immagini sul grande schermo e che, non importa quanto le immagini possano apparire vicine attraverso lo zoom o i primi piani più estremi, non può mai essere colmata o chiusa. Essa deve essere sempre fermamente mantenuta perché sia possibile la visione. Il piacere dello spettatore dipende da questa distanza erotico-conoscitiva; lá fermezza, la fissità, il rigore e la consistency' della distanza - qualità inerenti allá scrittura di Calvino - ne sono componenti essenziali e funzionali.

La scrittura di Calvino duplica questo meccanismo spettatoriale cinematografico fedelmente, come in uno specchio: non solo la realtà è ritratta come uno spettacolo visto e visibile a distanza, ma la mancanza o eliminazione della distanza vengono rappresentati come offuscamento e dissoluzione della realtà stessa (e a volte come punto d'entrata in un'altra realtà, dove però si deve rapidamente ristabilire una qualche distanza prospettica). ${ }^{11}$ La tecnica narrativa classica del punto di vista, in Calvino viene esaltata e raffinata in senso cinematico. C'è sempre "colui che vede," uno scrutatore, uno spettatore di cui seguiamo lo sguardo nel (rin)tracciare il senso di una storia. Leggere Calvino è sempre come vedere un film: uno non può mai dimenticare che sta guardando (anche se sta leggendo), né Calvino vuole che lo dimentichiamo. Calvino aveva orrore delle narrative del flusso della coscienza, e soprattutto odiava lo stile della "école du regard", dove la distanza (e la differenza) tra soggetto e oggetto, colui che guarda e ciò che viene guardato, sono ambigui e confusi.

Stephen Heath ha persuasivamente mostrato che la distanza strutturale inerente alla fenomenologia spettatoriale del cinema è legata alla scopofilia, e quindi alla paura della castrazione (cfr. "Difference", 51-112). Nel caso di Calvino questo meccanismo è ulteriormente complicato dalla femminizzazione e rimozione della voce: benché il canto delle sirene quando emerge dalla sala cinematografica sia infinitamente seducente, le sirene rappresentano pur sempre un pericolo mortale e devono essere messe a tacere e allontanate. L'immaginazione di Calvino e il suo modo di essere e scrivere da spettatore sembrano quindi essere radicati nelleconomia voyeuristica del cinemá narrativo di tipo hollywoodiano e nel principio del piacere visivo dello schermo (basato sulla logica della scopofilia) identificati da alcune 
teoriche femministe del cinema, e soprattutto da Laura Mulvey (Vedi "Visual Pleasure and Narrative Cinema" e "Afterthoughts on Visual Pleasure and Narrative Cinema" in Visual and Other Pleasures). Voyeuristica, ma anche narcisistica e feticistica, poiché queste tre dimensioni sono sempre congiunte. Narciso che si innamora dell'immagine riflessa su cui proietta il proprio desiderio è un mito archetipo del cinema, e la morte a cui Narciso va incontro per non aver mantenuto la distanza necessaria dall'immagine feticizzata conferma l'ineluttabile logica della fenomenologia spettatoriale. Come quella di Calvino, la fissazione di Narciso per e nell' immagine riduce a silenzio la voce femminile. Ipnotizzato dall'immagine di se stesso, Narciso diventa sordo alla voce di Eco come Calvino lo è a quella delle sirene una volta che è secluto nel cinema e guarda lo schermo ignorando il parlato. Come la ninfa Eco, che si dice fosse capace soltanto di ripetere, deformandole, le parole altrui, le voci dei doppiatori nel saggio autobiografico di Calvino tradiscono l'originale e non devono essere ascoltate. All'origine del sospetto, della paura e persino clellodio di Calvino per la parola parlata (tradizionalmente associata alla ingannevole seduzione femminile), è la paura del tradimento e dell'incoato e primitivo. La mollezza e mancanza di forma della parola ("informe, molle molle") ne fanno in effetti una sineddoche della femminilità. Solo la parola scritta e le immagini che essa costruisce e mette in moto sono dure, affidabili, e precise.

Il narcisismo di Calvino permea tutti i suoi testi creativi e critici anche quando (ed è quasi sempre) egli rifiuta di parlare di sé, cli dire "io", rifiuta cioè la dimensione autobiografica e introspettiva. Ciò che egli ci dà è però in effetti sempre un riflesso abbagliante e affascinante di se stesso e del suo immaginario come proiettato su un grande schermo o in uno specchio. In questo senso il titolo del saggio "Autobiografia di uno spettatore" (che avrebbe dovuto essere un saggio su Fellini, ma che di Fellini dice molto poco) è ridondante. Autobiografia e condizione di spettatore sono la stessa cosa per Calvino. Come lettori di Calvino ci troviamo sempre quindi nella posizione di voyeurs: guardiamo "Calvino" che guarda un' immagine riflessa di sé. Dico "noi" ma per una donna si tratta pur sempre di una posizione piuttosto difficile cla assumere: una lettrice di Calvino è sempre in qualche modo fuori posto, o meglio "spiazzata" dal lettore ideale di Calvino, il quale (con la parziale eccezione di Se uma notte d'imemo m viaggiatore, in cui con palese sforzo Calvino cerca di creare un ruolo e uno spazio anche per uná lettrice), è sempre implicitamente di sesso maschile.

la sala cinematografica è il luogo primario per un rito di iniziazione - l' iniziazione alla condizione di spettatore - in cui si pongono le basi per un'e- 
laborazione mitica, quella dello spettatore voyeurista-narcisista, con la sua distanzá necessaria dállo schermo. Nessuno ha descritto questo tipo di distánzá meglio di Roland Barthes in "En sortant du cinéma", un saggio sulla propria esperienzá di cinépbile scritto solo quattro anni dopo "Lautobiografia di uno spettatore" di Calvino. Secondo Barthes, una volta che il senso della "obbligatoria" distanza critica e ideologica dal film che si sta guardando viene messo tra parentesi, si può cominciare ad avere del cinema un esperienza diversa di "cloppia seduzione" che eccede e sorpassa il film. e che ha a che vedere con la situazione, la condizione stessa dello spettátore in quanto tale, secluto nel buio del cinematografo. Il primo aspetto di questa seduzione deriva dal fascino dello schermo, dove il corpo narcisistico s’immagina riflesso e si perde. Laaltro è la feticizzazione non dell’immagine sullo schemo ma di ciò che lo circonda e lo distanzia - l'oscurità, il fascio di luce del proiettore, l'insegna luminosá che indica l'uscita. "Ciò di cui mi servo per prendere le clistanze rispetto all'immagine, ecco, in fin dei conti, ciò che mi affascina: sono ipnotizzato da una distanza e questa distanza non è critica (intellettuale); è se così si può dire, una distanza amorosa".12 Secondo la fenomenologia dello spettatore cinematografico di Barthes, lo spettatore nella sala cinematografica non regredisce a una condizione rassicurante di oscurità intrauterina che gli permette di vivere indisturbato le proprie fantasie. Al contrario, egli deriva piacere dalla distanza stessa che lo separa dalle immagini sullo schermo, e clalla coscienza di essere in un cinematografo. La poetica della clistanza cinematografica di Calvino vive dello stesso erotismo. Invece di essere il sintomo di una lontananza puramente intellettuale e critical (la fredda razionalità che tanti critici gli rimproverano), la distanza di Calvino è come quella di Barthes, una distanza amorosá.

Non bisogna creclere però che Calvino amasse tutto il cinema. E' vero invece che egli amò molto "andare al cinema", l'esperienza del cinema. Calvino mantenne sempre un rapporto dilettantesco e amatoriale con il cinema. scrivendo occasionalmente recensioni e persino alcuni trattamenti e abbozzi di sceneggiature. Contrariamente a molti altri scrittori suoi contemporanei non entrò però mai professionalmente nel mondo del cinema. Questa condizione amatoriale fu proprio quella che gli permise di continuare ad amare il cinema: anche in questo sentí che era necessario mantenere una distanza, lá clistanzá necessaria a nutrire il desiderio. Ciononostante, Calvino aveva delle preferenze e dei gusti precisi in materia di cinema. Abbiamo già visto che cosa pensava dei film usciti durante l'era fáscista. Benché il suo fosse uno sguardo cinematografico e la sua narrativa esplo- 
rasse strategie analoghe a quelle cinematografiche, Calvino non ebbe una relazione facile col mondo del cinema dopo la seconda guerra mondiale, e con il tipo di spettatore "costruito" e sfruttato da tale cinema. Un articolo pubblicato su Cinema Nuovo (II, 10, 1 maggio 1953), intitolato "Il realismo italiano nel cinema e nella narrativa" è particolarmente rivelatore. Calvino fa riferimento all'esperienza "di massa" che andare al cinema era diventato in quegli anni: andare al cinema voleva dire "sedersi in mezzo a una platea di gente che sbuffa, ansima, sghignazza, succhia caramelle, ti disturba, entra, esce, magari legge le didascalie forte come al tempo del muto; il cinema è questa gente, più una storia che succede sullo schermo".13 Per quanto Calvino desideri sembrare interessato a questo tipo di esperienza di massa e ai film che la costruiscono e la sfruttano (come quelli di Steno e Monicelli e quelli di Luigi Zampa a cui fa riferimento l'articolo) è chiaro che il modello spettatoriale di Calvino è molto diverso ed è essenzialmente ancora quello delle sue prime esperienze a San Remo nel cinema vuoto in cui lui stava solo a "leggere" le immagini sullo schermo: "Forse sto invecchiando: vedo i film con lo stesso atteggiamento con cui leggo i manoscritti del mio ufficio in casa editrice. A pensarci è una cosa terribile: come mo che se na sta solo e legge"(ibid., p. 1889). Il cinema per Calvino è sempre stato, come la letteratura, un oggetto (per quanto amoroso e visivo), di lettura, e quindi un'esperienza essenzialmente solitaria e silenziosa. Il fatto che molti dei registi e sceneggiatori neorealisti fossero suoi amici, gente con cui parlava e andava spesso in trattoria, gli rendeva difficile guardare e amare i loro film. Il senso di meraviglia e di distanzá amorosa non c'erano piú, minati dalla familiarità. 14

Contrariamente a molti scrittori italiani della sua generazione per cui la narrativa neorealista e il cinema andavano naturalmente insieme, e che si muovevano con agio dall'una all'altro, Calvino scrisse un solo trattamento cinematografico di impianto neorealista (mai realizzato) ed era un critico cinemátografico reticente e spesso annoiato (uno dei suoi articoli scritti dal festival di Venezia si intitola "La noia a Venezia"). Pochissimi dei suoi testi sono stati adattati per il grande schermo, e con risultati che lasciano spesso a desiderare. ${ }^{15}$ Il cinema per Calvino rimase essenzialmente il cinema narrativo di Hollywood degli anni '30 che vicle da adolescente e da giovane prima della guerra. Calvino apprezzava anche a quellepoca il cinema francese e Jean Gabin era un suo idolo. Quasi tutto quello che venne dopo, dal neorealismo allá nomelle lague gli sembrò (con l'eccezione dei Western all'italiana di Sergio Leone, e di alcuni film di Nanni Moretti e di pochi altri) vuoto e insignificante. Particolarmente sgradito gli fu $A$ bout de souffle di Godard. 
Quando alla fine degli anni 60 e negli anni 70 a Parigi Calvino ricominciò ad andare al cinema quasi tutti i giorni, fu per vedere finalmente i film hollywoodiani degli anni '20 e '30 in versione originale e. sopratttutto, per ricordare e rivivere da lontano l’esperienza di spettatore dellá sua adolescenza. "Distanza" è di nuovo la parola chiave. Secondo quanto Cálvino ci dice in "Autobiografia di uno spettatore", i film di Hollywood lo aflascinavano in parte perché dispiegavano sullo schermo un mondo strano ed irreale di immagini in bianco e nero, corpi, visi, storie, situazioni e personaggi che non avrebbero potuto essere più Jontani dalla sua vita giornaliera a San Remo. Il cinema hollywoodiano aveva rappresentato per Calvino il primo incontro con forme di straniamento e defamiliarizzazione, che, trasformate in nuove strategie narrative e rappresentative, sarebbero poi diventate caratteristiche della sua opera. ${ }^{16}$ Con la precisione maniacale che avrebbe poi caratterizzato il suo modo di scrivere, Calvino si impadroní clel linguaggio del cinema hollywoodiano. Non solo imparò tutti i nomi degli attori e delle attrici, dei registi, dei produttori, e degli studios, ma si lasciò affascinare da quello che in effetti era il sistema semiotico astratto e convenzionale del cinema hollywoodiano, la codificazione rigorosa dei ruoli, dei generi, delle situazioni e delle connotazioni visive che, benché costituissero un sistema chiuso, permettevano infinite permutazioni. ${ }^{-}$L'esperienza fondatrice clell' andare al cinema segnò quindi l'inizio della sua ossessione per le permutazioni semiotiche, un'ossessione che eventualmente lo portò a opere combinatorie come Il castello dei destini incrociati e Se una notte d'imerno un viaggiatore. La rivisitazione dei film di Hollywood a Parigi contribui a compredere e a razionalizzare a posteriori la struttura combinatoria di quel cinema, ormai divenuto classico, che lo aveva cosi seclotto, e a attivare l'immaginazione semiotica del Calvino degli ultimi anni 60 e degli anni 70 e 80 .

I musicals di Fred Astaire e Ginger Rogers erano trai preferiti di Calvino. Come disse nell'intervista del 1981 con Lietta Tornabuoni, egli trovava che fossero meccanismi perfetti e formalmente conchiusi cli grande rigore e bellezza, mentre Fred Astaire era addirittura oggetto di adorazione da parte di Calvino: "le sue straordinarie doti di ballerino lo facevano appartenere a un mondo superumano, ma come personaggio umano aveva humor e grazia" (Pellizzari, cit., p. 131). Come clanzatore Fred Astaire è un esempio di qualità sempre ammirate da Calvino (che Pavese aveva battezzato "scoiattolo della penna"): leggerezza, agilità, rapiclità. L'incapacità di Calvino di creare un personaggio femminile di qualche profondità o interesse. $e$ in fondo l'indifferenzá di Cálvino nei confronti clel personaggio in gencrale e 
in particolare degli aspetti psicosessuali e fisici del desiderio e dell'erotismo sono legati alla superficialità cronica del cinema hollywoodiano (rappresentata in modo emblematico seppure elegantissimo dai musicals di Fred Astaire e Ginger Rogers) e alla tendenza di quel cinema a sublimare e purificare la sessualità. Quando Hollywood dovette piegarsi ai requisiti "morali" del codice Hays nel 1934, Fred Astaire non ebbe affatto bisogno di cambiare stile; la sua eleganza un po' asettica era già a prova di censore, e fu lui a promuovere e elevare Ginger Rogers (dal ruolo un po' troppo terrestre di Anytime Annie in 42 nd Street) a quello di sua eterna e eterea partner romantica. Come Calvino nota in "Autobiografia di uno spettatore", le donne erano dei fantasmi erotici extraterrestri nei film hollywoodiani, benché per ironia, intelligenza, risolutezza e competitività, le clonne incarnate da Katherine Hebpurn e soprattutto da Myrna Loy fossero perturbanti ed attraenti per il giovane Calvino, e divenissero una specie di ideale erotico che aveva il vantaggio di essere enormemente distante, rimosso in modo quasi assoluto dalla realtà delle donne in Italia in era fascista. ${ }^{18}$

1 Il testo si trova ora in Cakino, Romanzi e raccomli, 390-396.

2 Cfr. "Il senso della vista", in Laventura di uno spettatore. Italo Calvino e il cinema, 21-35. Costa sostiene che Palomar in particolare è una ricapitolazione della storia delle tecniche cinematografiche a partire adclirittura da antecedenti storici come la cronofotografia di Etienne-Jules Marey. Questultima ossevazione è particolarmente interessante perché fa pensare a un possibile legame tra Calvino e il futurismo.

3 Il saggio fu pubblicato come prefazione al libro di Federico Fellini, Quatro Film, e si trova ora in Romanzi e racconti, 27-49.

+ Il pezzo, che è una recensione dei film San Giolamini decollato e Alessandro, sei grande, è stato attribuito a Calvino da Pietro Ferrua. Cfr. Mondello 28. Ina bibliografia parziale di scritti di Calvino sul cinema si trova in Pellizzari, L'anentura, cit., 145-77.

5 Lat sceneggiatura, incitolata llarco Polo si può ora leggere nel terzo volume dei Romanzi e Raccomli di Calvino. 509-586. Nel suggerire quanto di profondamente cinematogralico ci sia nell'immaginario di Calvino, non intendo però suggerire che per lui la letteratura fosse meno importante. A questo proposito cfr. il mio "Calvino and the Value of Literature"

6 sul rema della distanza nella storia del rapporto di Calvino col cinema, cfr. Michele Canosa, "Lá distanzal" in Pellizzari, 49-67. Canosa traccia una cronaca delle variazioni del rema dedla distanza negli scritti di Cakino sul cinema ma ne trascura il ruolo strutturante nella poetica calviniana, e gli aspetti che hanno a che vedere con i rapporti di genere e las distanziazione del femminike.

Ciononostante o proprio per questo Calvino elbe una grande ammirazione per Chaplin, come si può dedure dalla sua recensione entusiastica di Monsieur Verdonx, 
"Chatlot e i finti tonti", pubblicata con il titolo "Chatot ha perso la pazicnzal" in Pemtite e" massa, aprile 19-18, 4- e ora in Sergeg, vol. 2, 1879-1882. In un articolo publalicato sull" Lniti il 31 maggio 1953, Calvino paragona inoltre Chaplin a Picasso: "Chaplin e l’icassos. i due più fantasiosi innamorati della vita che il nostro secolo comoscat. ci insegnano la fiducia nella vittoria dell’intelligenzal e della generosità umana" (Saggi, 2163).

8 Lintervista usci su La Stampa. 23 agosto 1981, a p. 3. ed è riprodotta in Pellizzari. $123-134$.

9 Il colloquio appante in Ferdinando Camon. Il mestiere di scrittore. Combrarami critiche poi in Calrino, Saggi, rol 2, 2-73-2-96. La frase citalla is a p. $2^{-76 .}$

$10 \mathrm{Mi}$ riferisco in paticolare ai Tresaggi sulla teoria della sessualità del 1905

11 In questo senso è da vedere per esempio il brano sulla "decostruzione" dellat scacchiera attaverso un ravicinamento prospettico progressivo e un tuffo nella porosita della materia lignea in Le cilli imeisibili.

12 Il saggio di Barthes ("En sortant du cinéma") usci su Commmmications e se ne (सb) una traduzione italiana qualche anno dopo.

13 1. articolo si trova orat riprodotto in Saggi, cit., 1888-1890.

14 Nello stesso aticolo del 1953. "Il realismo italiano nel cinema e nella narrativa," Calvino ossenal: "io trovo che vedere i film ha perso molto del suo carattere meraviglioso. adesso che quelli che li fanno sono i nostri amici . . . e mi diverto di meno." Si veda anche lintervista già citata del 1981 con Lietta Tomabuoni in Pellizzari, L atemmma di uno spellatore, 133, dove Calvino riconosce (pur con la sua usuale reticenza) l'impatto fondamentale della sue prime esperienze come spettatore: "I gusti delladolescenzal sono stati spazzati vial . . Però, chissà: non so. Quando sono entrato a far parte del mondo della carta stampita, il cinema fatto dal persone che potevo conoscere non mi faceral più tanta impressione. Non cera pin il semtimento di distanza. di mistero mitico. di dilatazione dei confini del reale" (corsiro mio).

15 Carlo di Carlo è autore di due film per la televisione, uno basato su $T$ con zero e uno su "Lavrentura di un lettore" da Gli amori difficili. C'è anche un adattamento televisivo del 1970 di Marcotaldo con la regia di Giuseppe Bennati. Altri tre racconti da Gli amori difficili sono stati trasformati in episodi di film: "Lavrentura di due sposi" è diventáto un episodio di Boccaccio 70 di Monicelli (1961) e "Lavientura di un soldato" un episodio di L'amore difficile di Vino Manfredi (1962). Francesco Maselli ha fatto un film a 16mm per la televisione basato su "L’alventura di un fotografo" (1982). C'è anche un adattamento parzialmente animato di $l l$ cavalieve inesistemte, realizzato da Pino Zatc nel 1969. Per una filmografia calviniana, cfr. Pellizzari, Lan'enmara, cit., 121-130. Nello stesso volume è da vedere lesilarante e borgesiano articolo di Pellizzari, "Se una notte d"inverno un produttore". sui film che si sarebbe potuto tratre dai litri di Calvino. "Futo in una pasticceria" uno dei primissini racconti di Calvino, è servito di ispirazione più o meno diretta a ben tre film molto bẹlli: I soliti ignoti di Mario Monicelli, Crackers di Louis Malle. e Palookarille di Alan Taylor.

16 Sulle tecniche di stranianmento in Cillvino. clr. il mio Calvino and the Age of Veorealism: Fables of Estrangement.

1" Nell'articolo già citato del 1953. "Il realismo italiano nel cinena e nella narrativa." prima della nascita della semiotica in Italia, Calvino (che però areva già letto Propp) discute i film hollymoodiano in termini che sono proto-semiotici: "ll mio cinema idealle . . resta quello americano dellanteguera, col suo cattalogo di clivi-personaggi, di convenzioni-situazioni. . quei film mi divertivo a vederli, e mi diventivo ancoral di più at rilletterci sopra, a smontarli, a demolirli . . anche quelli brutti erano interessanti e intruttivi. [Quel cinema] . . . averal creato un suo linguaggio - e una sual retorical - atutonomi."

18 I film hollywoodiani tutavia facevano naturalmente parte, una parte fondamentale, dellimmaginario delle donne italiane sotto il fascismo. 
OPERE CITATE

AA.VV. L'autentura di uno spettatore. Italo Calvino e il cinema. A cura di Lorenzo Pellizzari. Bergamo: Lubrina Editore, 1990.

Barthes, Roland. "En sortant du cinéma". Communications 23 (1975). Trad. italiana in AA.V Leggere il cinema. Milano: Mondadori, 1978.

Calvino, Italo. Romanzi e racconti. A cura di Mario Barenghi e Bruno Falcetto. Mondadori: Milano, 1994.

"Charlot ha perso la pazienza". In Id.Saggi. A cura. di Mario Barenghi.

Milano: Mondadori 1995, vol. 2, 1879-1882.

Camon. Ferdinando Il mestiere di scrittore. Comversazioni critiche. Milano: Garzanti, 1973.

Federico Fellini. Quattro Film. Einaudi: Torino 1974.

Heath, Stephen. “Difference”. Screen 19, 3 (1978): 51-112.

Mondello, Elisabetta. Italo Cal'ino, Edizioni Studio Tesi: Pordenone, 1990

Mulvey, Laura. risual and Other Pleasures. Bloomington: Indiana UP, 1989.

Re, Lucia. "Calvino and the Value of Literature". MLN 113 (1998): 121-137.

. Callino and the Age of Veorealism: Fables of Estrangement. Stanford: Stanford LP, 1990. 